\author{
${ }^{1 *}$ O. Bulgakova ${ }^{\circ},{ }^{1}$ A. Kıssainova! ${ }^{2} \mathrm{~N}$ Kalibekov ${ }^{\circ},{ }^{2}$ D. Serikbaiuly ${ }^{\text {, }}$ \\ ${ }^{3}$ T. Zinoveva , ${ }^{1}$ A. Aripova ${ }^{1}{ }^{1}$ R. Bersimbaev \\ ${ }^{1}$ L.N. Gumilyov Eurasian National University, Institute of Cell Biology and Biotechnology, Kazakhstan \\ ${ }^{2}$ National Research Oncology Center, Nur-Sultan, Kazakhstan \\ ${ }^{3}$ Multidisciplinary regional hospital of Akmola region, Kokshetau, Kazakhstan \\ *e-mail: ya.summer13@yandex.kz
}

\title{
The plasma levels of hsa-miR-19b-3p, hsa-miR-125b-5p and hsa-miR-155b-5p in NSCLC patients
}

\begin{abstract}
Lung cancer is one of the leading causes of cancer deaths worldwide. Presently for lung cancer patients various treatments are available, such as surgery, chemotherapy and radiotherapy. Nevertheless the 5 year overall survival remains still low. As many modern studies show, microRNAs can be novel and reliable biomarkers to predict the prognosis of lung cancer. Therefore, the aim of this study was to investigate the expression levels of hsa-miR-19b-3p, hsa-miR-125b-5p and hsa-miR-155b-5p in plasma of patients with non-small cell lung cancer (NSCLC) to clarify the relationships of free-circulating microRNAs expression with clinical factors and prognosis of NSCLC patients. Total RNA was extracted from blood samples of 49 NSCLC patients before treatment, 37 NSCLC patients after platinum-based chemotherapy and 50 healthy controls. The relative expression levels of microRNAs were evaluated by real time-polymerase chain reaction (RT-PCR). Kaplan-Meier method was used to analyze the survival curve. Plasma hsa-miR19b-3p, hsa-miR-125b-5p and hsa-miR-155b-5p expression levels in NSCLC patients were significantly upregulated compared with those in healthy individuals (all $\mathrm{P}<=0.01$ ). In plasma of NSCLC patients the expression level of hsa-miR-155b-5p significantly decreased in response to chemotherapy $(\mathrm{P}<0.01)$. NSCLC patients with high plasma hsa-miR-19b-3p and hsa-miR-155b-5p expression levels had a shorter overall survival than patients with low expression levels of these microRNAs $(P=0.005)$. Thus, the freecirculating hsa-miR-19b-3p and hsa-miR-155b-5p levels may serve as promising prognostic biomarkers in patients with NSCLC and hsa-miR-155b-5p can be used as biomarker for monitoring of effectiveness of antitumor therapy.
\end{abstract}

Key words: free-circulating microRNA, non-small cell lung cancer, hsa-miR-19b-3p, hsa-miR-125b-5p, hsa-miR-155b-5p, overall survival.

\section{Introduction}

MicroRNAs are a class of small non-coding endogenous RNAs with a length of 21-25 nucleotides that play an important role in the post-transcriptional regulation of gene expression by targeting mRNA followed by translation repression. MicroRNAs are involved in the pathogenesis of numerous diseases, including autoimmune, neurodegenerative diseases and cancer. Recently, the study of the level of freecirculating miRNAs has an increasingly widespread practical application in the diagnosis, prevention and treatment of various diseases [1]. Many studies have shown that microRNAs participate in tumorigenesis and progression of various types of cancer, including lung $[2 ; 3]$. Lung cancer is one of the leading causes of cancer deaths worldwide [4]. Unfortunately, to date, $100 \%$ sensitive and specific biomarkers for lung cancer have not been found. Moreover, some cancer-specific biomarkers were also found in plasma of healthy people [5]. Perhaps the development of microRNA-based panels would be effective for the early diagnosis of lung cancer.

Therefore, the purpose of this study was to assess the predictive value of free-circulating microRNAs hsa-miR-19b-3p, hsa-miR-125b-5p and hsa-miR$155 b-5 p$ for diagnosis, prognosis and effectiveness of antitumor therapy in NSCLC patients.

\section{Materials and methods}

Patients. A total of 86 blood samples of NSCLC patients and 50 blood samples of healthy individuals collected between 2015 and 2018 from Astana On- 
cology Center and Akmola region Oncology Hospital. All cases were newly diagnosed, previously untreated and histologically confirmed. Clinical stage was classified according to the sixth edition of the tumor-node-metastases (TNM) classification of the International Union Against Cancer.

49 samples were obtained before anticancer treatment, including surgery, chemotherapy and radiotherapy. 37 samples were obtained from patients after a course of platinum-based chemotherapy. The treatment was carried out in accordance with the Clinical Protocol for the Diagnosis and Treatment of Lung Cancer, recommended by the Expert Council of the Republican State Health Service at the Republican Center for the Development of Health Care of the Ministry of Health and Social Development of the Republic of Kazakhstan dated October 30, 2015 (Protocol No. 14) [6].

The study was approved by the Ethical Committee of the Astana Medical University, Kazakhstan (Astana, Kazakhstan; approval no. 4).

All participants were informed about the purpose and procedures, and their written informed were obtained.

RNA extraction from blood. A $10 \mathrm{ml}$ sample of whole peripheral blood was collected from each subject into ETDA containing tubes. Blood was centrifuged at 3,000 $\mathrm{xg}$ for $10 \mathrm{~min}$ and supernatant stored in aliquots at $-80^{\circ} \mathrm{C}$. Total RNA from $200 \mu \mathrm{L}$ plasma was isolated using the miRCURY RNA Isolation Kit - Biofluids (\#300112, EXIQON, Vedbaek, Denmark) in accordance with the protocol.

microRNAs analysis by $q P C R$. The expression levels of microRNAs were determined by evaluating the level of fluorescence emitted by SYBR GREEN tracer. MiRCURY LNA ${ }^{\mathrm{TM}}$ UNIVERSAL RT microRNA PCR LNA $^{\mathrm{TM}}$ kit, including microRNAs specific primers hsa-miR-19b-3p (\#204450, Exiqon, Denmark), hsa-miR-125b-5p(\#205713, Exiqon, Denmark), hsa-miR-155-5p(\#204308, Exiqon, Denmark) was used to amplify microRNAs according to the manufacturer's instructions. cDNAs were prepared using Universal synthesis Kit (\#203301, EXIQON, Vedbaek, Denmark) according to the manufacturer's instructions. Real-time PCR amplification was performed in the CFX96 Touch ${ }^{\mathrm{TM}}$ Real-Time PCR Detection System (Bio-Rad). Each reaction was carried out using $4 \mu \mathrm{L}$ of cDNA, $1 \mu \mathrm{L}$ of PCR primer mix and $5 \mu \mathrm{L}$ SYBR-Green PCR Master mix (\#203403, EXIQON, Vedbaek, Denmark) in a final $10 \mu \mathrm{L}$ volume. The qRT-PCR was performed at $95^{\circ} \mathrm{C}$ for 10 min for one cycle, then $95^{\circ} \mathrm{C}$ for $10 \mathrm{sec}$ with $60^{\circ} \mathrm{C}$ for $60 \mathrm{sec}$ for 40 cycles. Gene expression was nor-

Int. j. biol. chem. (Online) malized to RNU6B (\#203907, EXIQON, Vedbaek, Denmark). All reactions were carried out in triplicate, and the $2^{-\Delta \Delta C t}$ method was used to quantify the relative microRNA amount [7].

Statistical Analysis. Differences in microRNA levels between NSCLC patients and healthy control were compared using a paired Student's t-test. Kaplan-Meier method was used to analyze the survival curve. $P$ value $<0.05$ considered as significant. These statistical analyses were performed using GraphPad Prism 7.0 (Graphpad software Inc., CA, USA).

\section{Results and discussion}

Study design. The study included 50 volunteers without pulmonary pathologies and acute/chronic inflammatory diseases (control), among them 11 women and 39 men. The average age in the group was 47 years. The group consisted of 8 smokers, 41 non-smokers and 1 former smoker. The group of lung cancer patients included 86 people, among them 16 women and 70 men. The average age in the group was 65 years. The lung patients group consisted of 34 smokers, 33 non-smokers and 19 former smokers.

The group of lung cancer was consisted of 20 patients with pathologic stage I NSCLC, 32 patients with stage II, 25 patients with stage III and 9 patients with pathologic stage IV NSCLC.

Comparison of two groups ("Lung cancer" and "Control") by sex, age and smoking status presented in Table 1.

Table 1 - Characteristics of the subject participating in the study

\begin{tabular}{|c|c|c|c|}
\hline & $\begin{array}{l}\text { Lung cancer } \\
\quad(\mathrm{n}=86)\end{array}$ & $\begin{array}{l}\text { Control } \\
(\mathrm{n}=50)\end{array}$ & $\mathrm{P}$ \\
\hline Male & $70(81 \%)$ & $39(78 \%)$ & \multirow{2}{*}{0.12} \\
\hline Female & $16(19 \%)$ & $11(22 \%)$ & \\
\hline Age $\leq 60$ & $30(35 \%)$ & $21(42 \%)$ & \multirow{2}{*}{0.35} \\
\hline Age $>60$ & $56(65 \%)$ & $29(58 \%)$ & \\
\hline Non-smokers & $33(34 \%)$ & $41(82 \%)$ & \multirow{3}{*}{0.36} \\
\hline Smokers & $34(39 \%)$ & $8(16 \%)$ & \\
\hline Former Smokers & $19(27 \%)$ & $1(2 \%)$ & \\
\hline
\end{tabular}

NSCLC patients were divided into two experimental groups: lung cancer before treatment and lung cancer after treatment, depending on the presence/absence of chemotherapy. The first group consisted of 49 people; the second group consisted of 37 people. 
Comparison of the experimental groups "Lung cancer after treatment" and "Lung cancer before treatment" are shown in Table 2.

Table 2 - Comparative characteristic of the experimental groups "Lung cancer before treatment" and "Lung cancer after treatment"

\begin{tabular}{|c|c|c|c|}
\hline & $\begin{array}{c}\text { Lung cancer } \\
\text { after treatment } \\
(\mathrm{n}=37)\end{array}$ & $\begin{array}{c}\text { Lung cancer } \\
\text { before treatment } \\
(\mathrm{n}=49)\end{array}$ & $\mathrm{P}$ \\
\cline { 1 - 3 } Male & $31(84 \%)$ & $39(80 \%)$ & \multirow{2}{*}{0.78} \\
\cline { 1 - 3 } Female & $6(16 \%)$ & $10(20 \%)$ & \multirow{2}{*}{0.51} \\
\cline { 1 - 3 } Age $\leq 60$ & $18(27 \%)$ & $14(8 \%)$ & \multirow{2}{*}{0.52} \\
\cline { 1 - 3 } Age $>60$ & $27(73 \%)$ & $45(92 \%)$ & \\
\cline { 1 - 3 } Non-smokers & $9(24 \%)$ & $24(49 \%)$ & \\
\cline { 1 - 3 } Smokers & $21(57 \%)$ & $13(27 \%)$ & \\
\cline { 1 - 3 } Former & $7(19 \%)$ & $12(24 \%)$ & \\
\hline Smokers & \multicolumn{2}{|c|}{} &
\end{tabular}

The level of relative expression of free-circulating microRNA hsa-miR-19b-3p in lung cancer patients

The results showed that the level of expression of hsa-miR-19b-3p in patients with lung cancer before treatment was 6.5 times higher than in healthy people ( $p<0.0001)$. Similar data were obtained by analyzing the expression of hsa-miR-19b-3p in the plasma samples of patients with lung cancer after treatment. In this group, the level of hsa-miR-19b-3p was 6.9

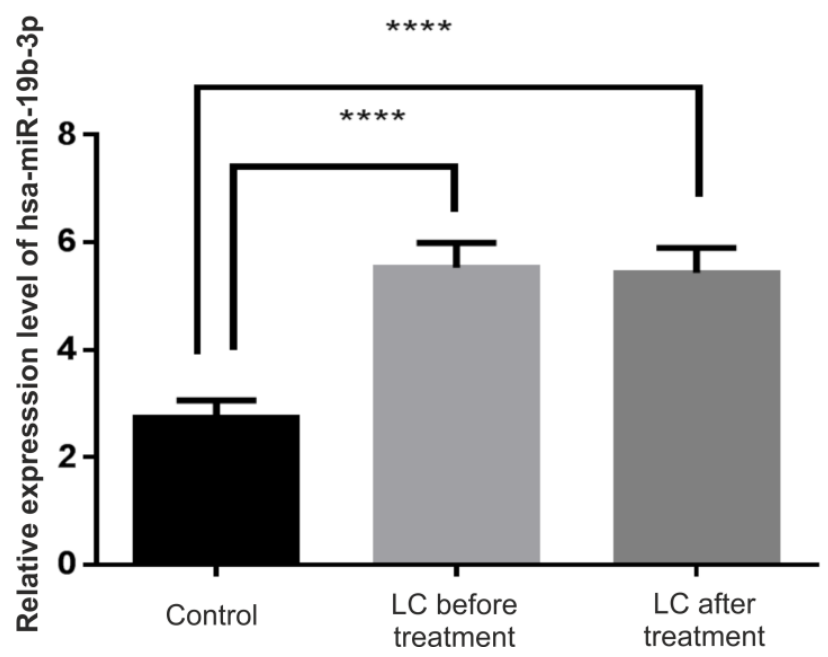

Figure 1 - Relative expression level of hsa-miR-19b-3p in patients with lung cancer before and after treatment compared to control times higher compared with the control $(\mathrm{p}<0.0001)$ (Figure 1). There was no difference in expression of hsa-miR-19b-3p in both groups of lung cancer patients.

The level of relative expression of free-circulating microRNA hsa-miR-125b-5p in lung cancer patients

The relative expression level of hsa-miR-125b$5 p$ in plasma samples of lung cancer patients before treatment was 4 times higher than that in plasma samples of healthy individuals $(p<0.001)$. The results showed no differences in the expression level of hsa-miR-125b-5p in plasma of lung cancer patients after treatment and healthy people from the control group. Comparison of the expression level of hsamiR-125b-5p in blood plasma of lung cancer patients showed that the expression level was almost 2 times lower after treatment than in lung cancer patients before treatment (Figure 2).

The level of relative expression of free-circulating microRNA hsa-miR-155-5p in lung cancer patients

In plasma of patients with lung cancer before treatment, the expression level of hsa-miR-155$5 \mathrm{p}$ was 2 times higher compared to the control group $(\mathrm{p}<0.001)$. Difference in the level of hsamiR-155-5p expression between the lung cancer patients after treatment and healthy individuals was not detected. A twofold decrease in the level of free-circulating hsa-miR-155-5p was observed in plasma samples of lung cancer patients after treatment compared to patients before treatment $(\mathrm{p}$ $<0.01$ ) (Figure 3).

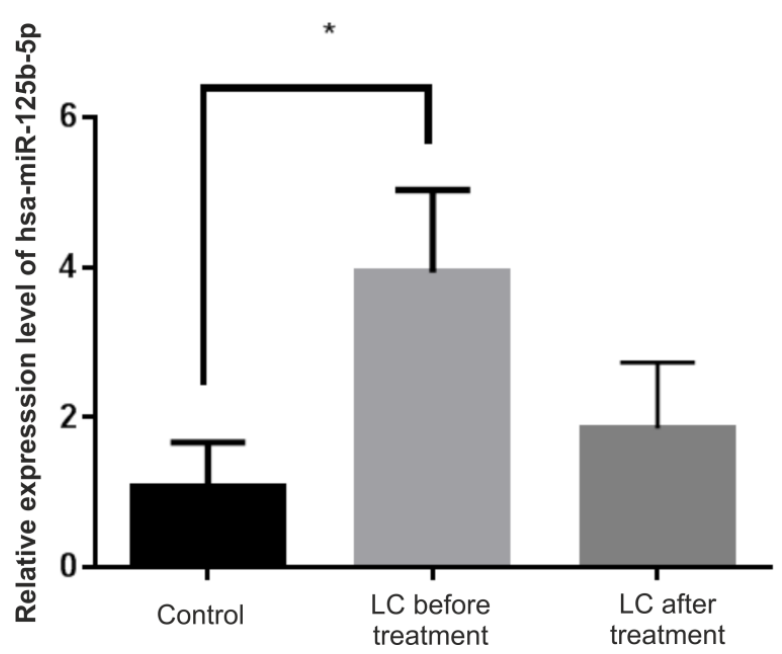

Figure 2 - Relative expression level of hsa-miR-125b-5p in lung cancer patients before and after treatment compared to control 


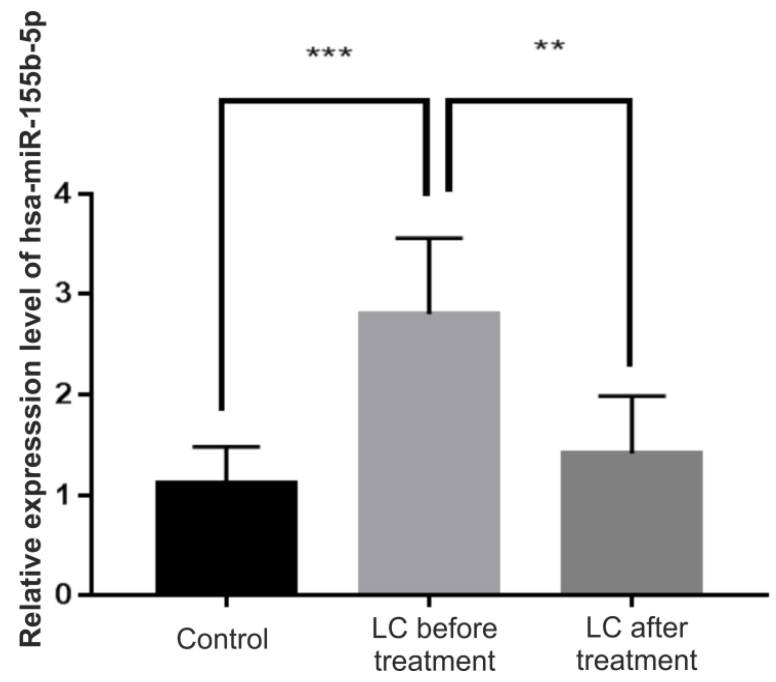

Figure 3 - Relative expression level of hsa-miR-155b-5p in lung cancer patients before and after therapy compared to control
Survival of lung cancer patients according to the expression levels of free-circulating microRNA hsamiR-19b-3p, hsa-miR-125b-5p, hsa-miR-155-5p

Univariate analysis revealed that patients with high expression of hsa-miR-19b-3p had shorter survival time than those with low expression $(\mathrm{HR}=$ 2.33, 95\% CI: 1.04-5.230, $\mathrm{p}=0.02$ ) (Figure 4A). Plasma level of hsa-miR-125b-5p was not associated with survival in lung cancer patients $(\mathrm{p}=0.2)$. High expression level of hsa-miR-155b-5p was significantly associated with worse survival in lung cancer patients $(\mathrm{HR}=2.78,95 \% \mathrm{CI}: 1.32-5.88, \mathrm{P}=0.005)$ (Figure 4D).

Then combination of plasma hsa-miR-19b-3p and hsa-miR-155b-5p was analyzed. Using the lowrisk group (low hsa-miR-19b-3p and hsa-miR-155b$5 \mathrm{p}$ expression) as a reference, patients with high hsamiR-19b-3p and hsa-miR-155b-5p expression had 2.14- (95\% CI: 1.147 to $4.092, \mathrm{p}=0.005)$ increased risk of death.

\section{$A$}

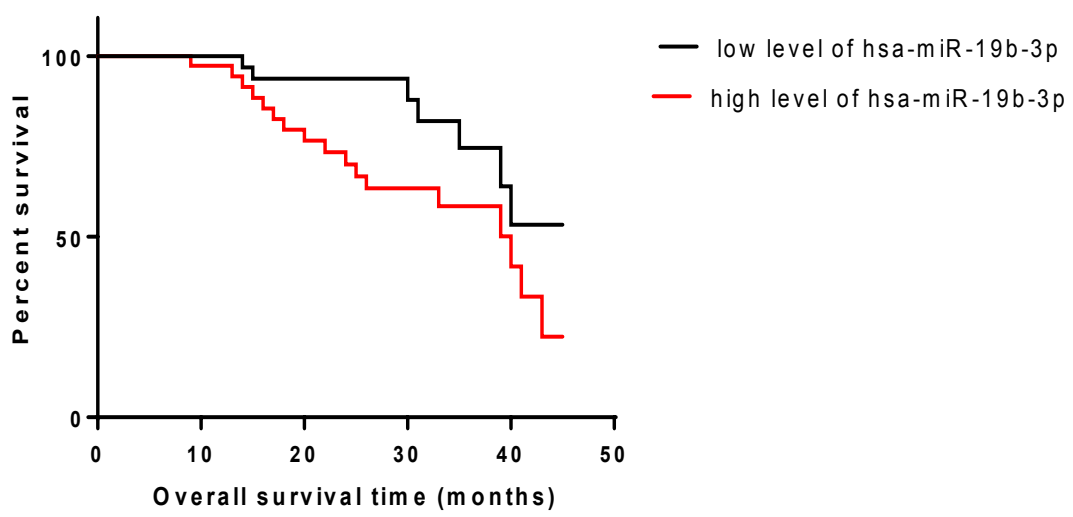

$B$

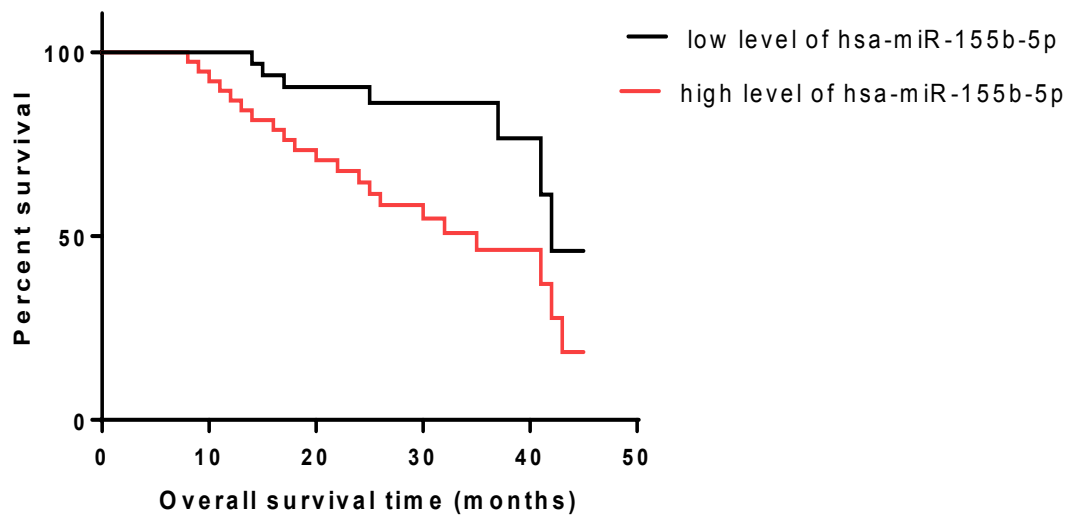

Figure 4 - Kaplan-Meier survival curves of lung cancer patients according to the expression levels of free-circulating microRNA hsa-miR-19b-3p (A) and hsa-miR-155-5p (B) 
Despite the accuracy of diagnostic methods used in modern pathology has dramatically improved, the early diagnosis of lung cancer remains challenging. Unique microRNA expression profiles have been observed in various cancer types at different stages, suggesting their potential as diagnostic and prognostic biomarkers. In addition, there is a requirement to new sensitive and specific prognostic markers which can be used to determine the effectiveness of chemotherapy and the individual response of the patient to the treatment.

microRNAs can modulate the expression of multiple genes, playing essential roles in many cellular processes, and thus microRNAs can directly influence the development and progression of many types of cancer [1]. Unique microRNA expression profiles have been observed in various cancer tumors [1], suggesting their potential as diagnostic and prognostic biomarkers. The miR-19 family (miR-19a and miR19b) are key oncogenic components of the miR-1792 cluster [8]. It was shown that miR-19b enhances the proliferation and apoptosis resistance of cancer cells by the epidermal growth factor receptor (EGFR) signaling pathway [9]. Moreover, overexpression of miR-19b observed in human cancer cells can diminish p53 protein levels [10].

In our study we have shown aberrant expression level of hsa-miR-19b-3p in plasma of NSCLC patient. Our data are consistent with other studies $[11 ; 12]$. Considering these results, hsa-miR-19b-3p might serve as an oncogene, and play a crucial role during tumor development of lung cancer. However, it is interesting that the expression of hsa-miR-19b$3 p$ remained at the same level in the group after platinum-based chemotherapy.

Based on the altered this microRNA expression in plasma of NSCLC patients, we further analyzed the clinical value of hsa-miR-19b-3p in lung cancer prognosis. According to the Kaplan-Meier survival analysis, we observed that patients with high hsa-miR-19b$3 \mathrm{p}$ expression had a shorter survival time than those with low expression, suggesting that overexpression of hsa-miR-19b-3p might be closely correlated with poor overall survival of lung cancer patients.

There are a lot of controversial data about role of hsa-miR-125b in human cancer. Thus, Gong et al. shown that miR-125b may promote apoptosis by suppressing the anti-apoptotic molecules of the Bcl-2 family [13]. One there over hand, it was demonstrated, that miR-125b is an important negative regulator of p53 and p53-induced apoptosis [14; 15]. There is the same situation in the question about a role of miR-125b as biomarkers for diagnosis of lung cancer.

Int. j. biol. chem. (Online)
Different studies have shown that miR-125b is upregulated [16] or down-regulated [17] in lung cancer. Our results are consistent with the data from other authors on the role of hsa-miR-125b as an oncomiR since over-expression of this microRNA is observed in plasma blood samples of lung cancer patients. The trend of down-regulation of hsa-miR-125b in NSCLC patients after treatment was found $(\mathrm{p}=0.06)$. There was no significant relationship between high expression levels of blood free-circulating hsa-miR$125 \mathrm{~b}$ and patient survival prognosis. $\mathrm{Li}$ et al. had shown that miR-125b promoted tumor metastasis in NSCLC patients [18]. In our study we could not find correlation between metastasis progression and the expression level of hsa-miR-125b (data not shown).

Many studies had shown that altered expression of miR-155 was associated with various physiological and pathological processes, including hematopoietic lineage differentiation [19], immune response [20], inflammation [21] and tumorigenesis [22]. Furthermore, Lv et al. [23] found that miR-155 drives therapy resistance mechanisms in human lung cancer A549/dox cells. Our results demonstrated overexpression of hsa-miR-155b-5p in blood plasma of NSCLC patients compared to control. Moreover, high hsa-miR-155b-5p expression level was found to be correlated with poor survival in lung cancer patients. Our data suggest that has-miR-155b-5p can be promising biomarker for monitoring of effectiveness of antitumor therapy. Because, the significant decrease in the level of free-circulating hsa-miR-155$5 \mathrm{p}$ was observed in NSCLC patients after treatment compared to patients before treatment. Our data are consistent with the results of Van Roosbroeck [24], who showed, that combined treatment of miR-155 inhibitor and cisplatin significantly reduced viability of lung cancer cells.

\section{Conclusion}

Present study showed that plasma levels of hsamiR-19b-3p, hsa-miR-125b-5p and hsa-miR-155b$5 \mathrm{p}$ were associated with lung cancer and could be useful for further development of microRNA-based panels for early diagnosis of NSCLC. Free-circulating hsa-miR-19b-3p and hsa-miR-155b-5p can be used as prognostic biomarkers for NSCLC patients. Plasma levels of hsa-miR-155b-5p may serve as biomarker for NSCLC patients receiving platinum-based chemotherapy. Further studies are needed to investigate the role of hsa-miR-125b-5p as biomarker for monitoring of effectiveness of antitumor therapy in NSCLC. 


\section{Acknowledgements}

We thank the Astana Oncology Center (Astana city) and Akmola Region Oncology Hospital (Kokshetau city) for their help in collection of samples.

\section{References}

1. Izzotti A., Carozzo S., Pulliero A., Zhabayeva D., Ravetti J.L., Bersimbaev R. (2016) Extracellular MicroRNA in liquid biopsy: applicability in cancer diagnosis and prevention. Am. J. Cancer Res., vol. 6, pp. 1461-1493.

2. Wu K.L., Tsai Y.M., Lien C.T., Kuo P.L., Hung A.J. (2019) The Roles of MicroRNA in Lung Cancer. Int. J. Mol. Sci., vol. 20, p. 1611.

3. Bulgakova O., Zhabayeva D., Kussainova A., Pulliero A., Izzotti A., Bersimbaev R. (2018) miR-19 in blood plasma reflects lung cancer occurrence but is not specifically associated with radon exposure. $\mathrm{On}$ col. Lett., vol. 15, pp. 8816-8824.

4. Siegel R., Ma J., Zou Z., Jemal A. (2014) Cancer statistics. CA Cancer J. Clin., vol. 64, pp. 9-29.

5. Zamay T.N., Zamay G.S., Kolovskaya O.S., et al. (2017) Current and Prospective Protein Biomarkers of Lung Cancer. Cancers, vol. 9, p. 155.

6. Kazakh Research Institute of Oncology and Radiology. https://onco.kz/klinicheskie-protokoladiagnostiki-i-lechenie-zlokachestvennyh-novoobrazovanij/

7. Livak and Schmittgen (2001) Analysis of relative gene expression data using real-time quantitative PCR and the 2- $\Delta \Delta \mathrm{Ct}$ method. Methods, vol. 25, pp. 402-408.

8. Zhang X., Li Y., Qi P., Ma Z. (2018) Biology of MiR-17-92 Cluster and Its Progress in Lung Cancer. Int J Med Sci., vol. 15, pp. 1443-1448.

9. Baumgartner U., Berger F., Hashemi Gheinani A., Burgener S.S., Monastyrskaya K., Vassella E. (2018) miR-19b enhances proliferation and apoptosis resistance via the EGFR signaling pathway by targeting PP2A and BIM in non-small cell lung cancer. Mol. Cancer, vol. 17, p. 44.

10. Fan Y., Yin S., Hao Y., Yang J., Zhang H., Sun C., Ma M., Chang Q., Xi J.J. (2014) miR-19b promotes tumor growth and metastasis via targeting TP53. RNA, vol. 20, pp. 765-772.

11. Zaporozhchenko I.A., Morozkin E.S., Skvortsova T.E., Ponomaryova A.A., Rykova E.Y., Cherdyntseva N.V., Polovnikov E.S., Pashkovskaya O.A., Pokushalov E.A., Vlassov V.V., Laktionov P.P. (2016) Plasma miR-19b and miR-183 as Potential Biomarkers of Lung Cancer. PLoS One, vol. 11, e0165261.

12. Lu S., Kong H., Hou Y., Ge D., Huang W., Ou J., Yang D., Zhang L., Wu G., Song Y., Zhang X., Zhai C., Wang Q., Zhu H., Wu Y., Bai C. (2018) Two

Int. j. biol. chem. (Online) plasma microRNA panels for diagnosis and subtype discrimination of lung cancer. Lung Cancer, vol. 123, pp. 44-51.

13. Gong J., Zhang J.P., Li B., Zeng C., You K., Chen M.X., Yuan Y., Zhuang S.M. (2013) MicroRNA-125b promotes apoptosis by regulating the expression of Mcl-1, Bcl-w and IL-6R. Oncogene, vol. 32, pp. 3071-3079.

14. Le M.T., Teh C., Shyh-Chang N., Xie H., Zhou B., Korzh V., Lodish H.F., Lim B. (2009) MicroRNA-125b is a novel negative regulator of $\mathrm{p} 53$. Genes Dev., vol. 23, pp. 862-876.

15. Yuxia M., Zhennan T., Wei Z. (2012) Circulating miR-125b is a novel biomarker for screening non-small-cell lung cancer and predicts poor prognosis. J. Cancer Res. Clin. Oncol., vol. 138, pp. 20452050

16. Zhang Y., Huang S. (2015) Up-regulation of miR-125b reverses epithelial-mesenchymal transition in paclitaxel-resistant lung cancer cells. Biol. Chem., doi: https://doi.org/10.1515/hsz-2015-0153.

17. Yu X., Wei F., Yu J., Zhao H., Jia L., Ye Y., Du R., Ren X., Li H. (2015) Matrix Metallopeptidease 13: A Potential Intermediate Between Low Expression of microRNA-125b and Increasing Metastatic Potential of Non-Small Cell Lung Cancer. Cancer Genet., vol. 208, pp. 76-84.

18. Li Q, Han Y, Wang C, Shan S, Wang Y, Zhang J, Ren T (2015) MicroRNA-125b promotes tumor metastasis through targeting tumor protein 53-induced nuclear protein 1 in patients with non-small-cell lung cancer. Cancer Cell Int., doi: 10.1186/s12935-015-0233-x.

19. Van Roosbroeck, K., Calin, G.A (2016) MicroRNAs in chronic lymphocytic leukemia: miRacle or miRage for prognosis and targeted therapies? Semin Oncol., vol. 43, pp. 209-214.

20. Alivernini S, Gremese E, McSharry C, Tolusso B, Ferraccioli G, McInnes IB, Kurowska-Stolarska M (2018) microRNA-155 - at the critical interface of innate and adaptive immunity in arthritis. Front. Immunol., doi: 10.3389/fimmu.2017.01932.

21. Mahesh G., Biswas R. (2019) MicroRNA-155: a master regulator of inflammation. $J$. Interferon Cytokine Res., vol. 39, pp. 321-330.

22. Chen S., et al. (2015) Host miR155 promotes tumor growth through a myeloid-derived suppressor cell-dependent mechanism. Cancer Res., vol. 75, pp. 519-531.

23. Lv L., et al. (2016) Effect ofmiR-155 knockdown on the reversal of doxorubicin resistance in human lung cancer A549/dox cells. Oncol Lett., vol. 11, pp. 1161-1166.

24. Van Roosbroeck, K., et al. (2017) Combining anti-Mir-155 with chemotherapy for the treatment of lung cancers. Clin Cancer Res., vol. 23, pp. 28912904. 